\title{
Three-dimensional structure-based approach for the analysis of macroscopic lead structures on sealing counterfaces
}

\author{
Maximilian Engelfried* (D), Matthias Baumann(D), and Frank Bauer(D) \\ University of Stuttgart, Institute of Machine Components (IMA), Stuttgart, Germany
}

Received: 8 September 2021 / Accepted: 12 January 2022

\begin{abstract}
The seal failure of an elastomer rotary shaft seal is often caused due to lead on the shaft counterface. In sealing technology, the term 'lead' includes all structures on sealing counterfaces that are capable of transporting fluid in axial direction through the sealing contact and thus disrupting the sealing mechanism. Lead structures are created during the manufacturing process of the shaft surface or throughout the handling. They occur in various shapes and sizes. Depending on the characteristics of the lead structures, several specialized measurement and evaluation methods exist which have to be applied in combination. However, not all types of lead can be covered with the methods known so far. State of the art are frequency-based and model-based analysis methods, which are only able to detect periodic lead structures. Aperiodic and stochastically distributed lead structures cannot be detected due to the functional principle. This article provides an approach for a structure-based evaluation of macroscopic lead structures based on optical topography measurement data. This allows to detect all known types of macroscopic lead on the shaft surface and in future to measure microscopic and macroscopic lead with a single measurement procedure.
\end{abstract}

Keywords: Optical topography measurements / lead measurement / structure-based analysis methods / sealing technology

\section{Introduction}

Elastomer rotary shaft seals are widely used to seal the passage of a rotating shaft and a housing. They are standardized machine elements according to [1-3]. The dynamic leak tightness of the sealing system is due to the formation of an active back-pumping effect of the rotary shaft seal. Here, the sealing edge floats on a fluid film during the rotation of the shaft, but the fluid does not leak through the sealing gap to the outside. The fluid in the sealing gap is constantly exchanged with fluid in the housing because of the back-pumping effect. More information about the physical mechanisms can be found in [4]. Optimally, the shaft surface does not interfere with the back-pumping fluid flow. However, if there are lead structures on the shaft surface, they cause the shaft to pump the fluid into or out of the sealing contact. Increased wear and leakage can result from it $[5,6]$.

Figure 1 shows how the sealing effect of the rotary shaft seal can be disturbed by lead structures. Besides the three main components of the rotary shaft seal, Figure 1

\footnotetext{
* Corresponding author:

maximilian.engelfried@ima.uni-stuttgart.de
}

illustrates a superimposition of the pumping flows of the rotary shaft seal and the sealing counterface.

The back-pumping flow of the rotary shaft seal is shown in green, the pumping flow of the sealing counterface in red. The amount and the direction of the sealing counterface's pumping flow depends on the lead structure's shape and its orientation in combination with the shaft's direction and speed of rotation [7]. The superposition of both fluid flows results in the yellow shown fluid flow and can have the following disturbing influences on the sealing mechanism. Leakage (failure mode 1) occurs when the pumping flow of the shaft is directed outwards and quantitatively greater than the back-pumping flow of the rotary shaft seal. Dry run (failure mode 2) due to insufficient lubrication is traced back to pumping flows both directed into the interior. This causes thermal damage to the sealing edge and, in the long run, leakage and seal failure.

In order to avoid lead and resulting seal failures, sealing counterfaces are subjected to quality analysis. Here, the shaft surface must be measured first. Then, lead structures must be localized on the topographic measurement data. There are already several measuring and analysis methods for the analysis of certain lead categories. Previously, it was assumed that macroscopic lead structures always appear periodically as a kind of thread on the shaft surface. 

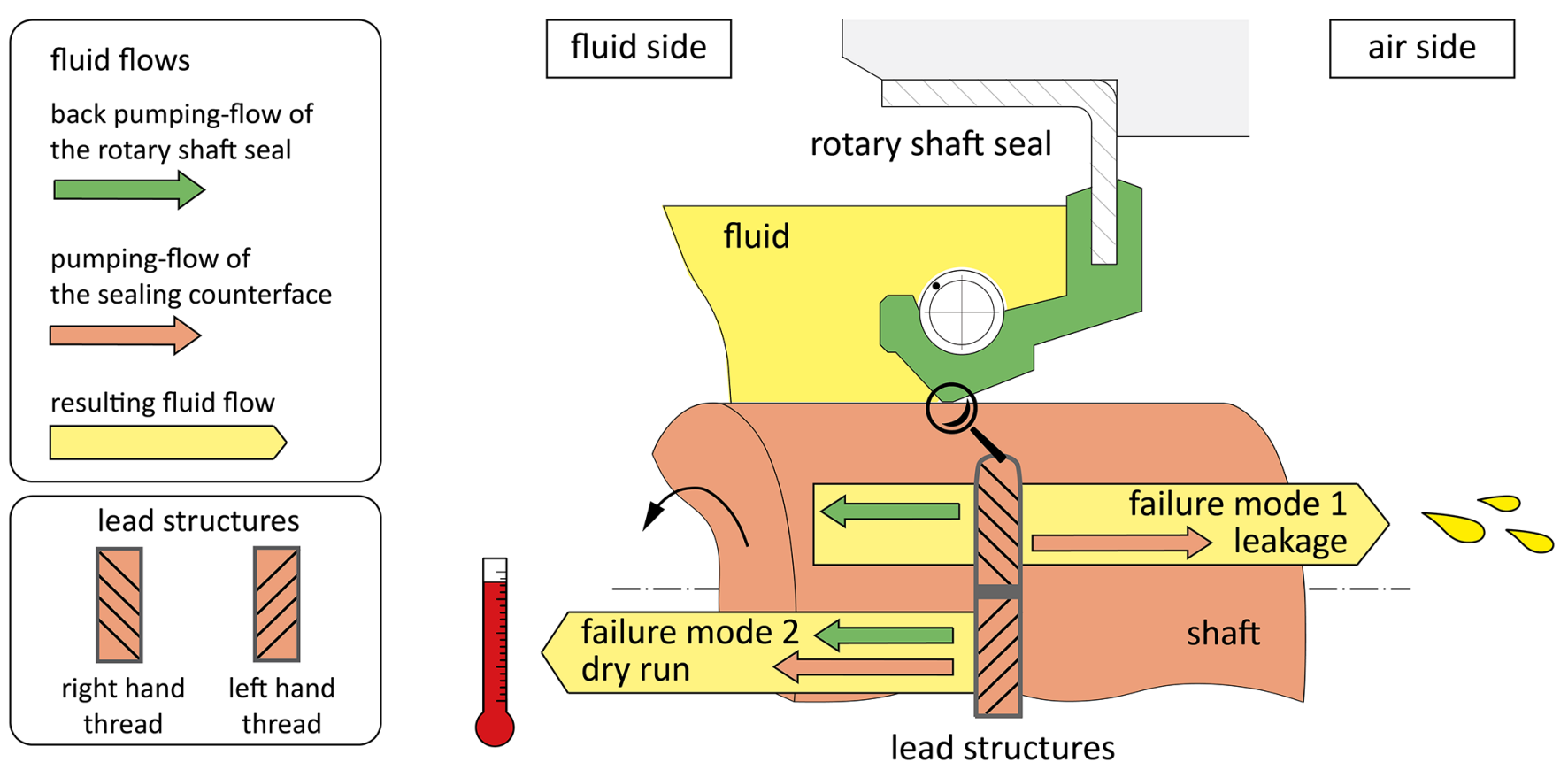

Fig. 1. Rotary shaft sealing and the impact of lead on the sealing mechanism.

Therefore, frequency-based methods have proven to be suitable. Further research in the field of sealing technology revealed that aperiodic and stochastic structures can also occur on sealing counterfaces and pump fluid. New analysis methods are required for the localization of these types of structures.

\section{Lead structures on sealing counterfaces - categorisation and measuring methods}

Lead structures can be classified depending on their size, shape and type of origin into micro lead, micro waviness, macro lead and defects. Apart from defects such as scratches that occur during handling, the formation of lead is caused by the manufacturing process. Stochastically arranged, anisotropic structures of microscopic size formed by the intervention of individual abrasive grains during the grinding process are referred to as the micro lead [8]. To localize them on the measured surface, a Gaussian highpass filter of cutoff wavelength $\lambda_{c}=20 \mu \mathrm{m}$ is used [9]. The cut-off wavelength was determined on the basis of the average width of the individual grinding grooves, which is around $15 \mu \mathrm{m}$ [7]. Macroscopic lead structures which are classified in terms of size between the micro lead and regular waviness ${ }^{1}$ on sealing counterfaces, are located in the topography resulting from Gaussian low-pass filtering. They are created during manufacture by transferring and superimposing the contour of the grinding wheel or other tools to the shaft surface [8]. One type of macroscopic surface structures occurs axially periodically. These structures circulate the shaft continuously in circumferential direction and are known as macro lead. Macro lead and

\footnotetext{
$\overline{1}>\lambda_{\mathrm{c}}=0,8 \mathrm{~mm}$ for typical roughness values on the counterface of rotary shaft seals according to ISO 4288 [10].
}

micro lead are described in the Mercedes-Benz company standard (MBN) 31007-7 [11]. But the standard does not consider aperiodic structures of macroscopic dimensions with varying shapes and orientations. These are becoming increasingly important with the testing of alternative manufactured sealing counterfaces. Current research results on functional behaviour can be found e.g. in $[12,13]$. Baitinger [9] and Baumann [7] introduced the term "micro waviness" to describe all types of macroscopic lead structures. Thus, macroscopic structures with non-periodic appearance can be also included in harmful structures on sealing counterfaces. Baumann further specifies macro lead with its periodic form as a special case of micro waviness, all other structures of the same size as macro lead receive the designation aperiodic micro waviness [7]. To simplify the terminology, in this paper the term "micro waviness" covers the aperiodic and stochastically distributed lead structures. Together with the macro lead, it forms the macroscopic lead structures that are the focus of this new structure-based evaluation approach. The microscopic structures, separated by Gaussian filtering with $\lambda_{c}=20 \mu \mathrm{m}$, are described solely by the micro lead. Figure 2 gives a classification of lead and an overview of common used analysis methods.

\subsection{Quantitative analysis of macro lead structures (according to MBN 31007-7)}

A widely used method for the quantitative analysis of macro lead on sealing counterfaces is the macro lead measurement according to MBN 31007-7, colloquially also called CARMEN-method (Computer Aided Roughness Measurement and Evaluation). Its measurement procedure, evaluation and macro lead parameters are standardized according to Mercedes-Benz company standard MBN 31007-7 [7]. The macro lead measurement according to 


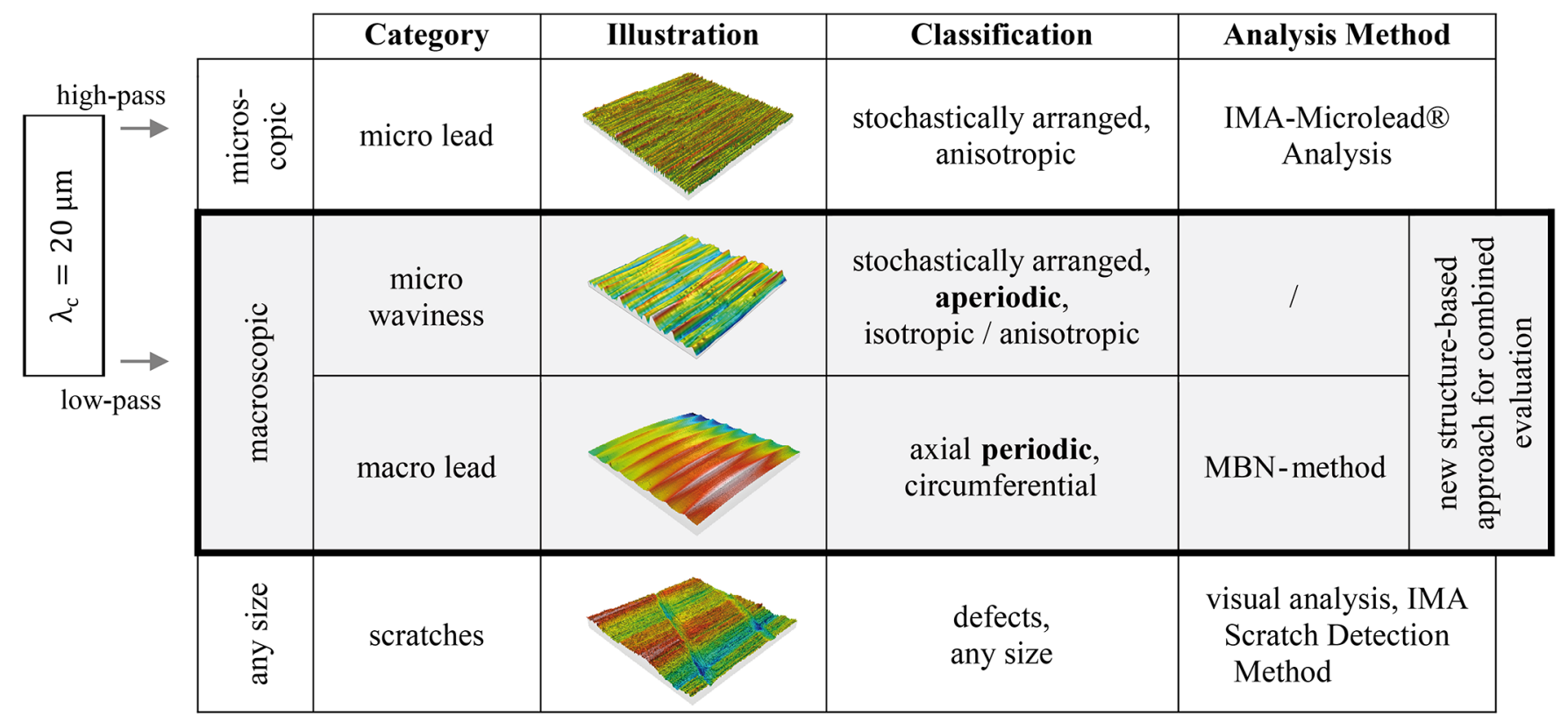

Fig. 2. Classification of lead structures on shaft counterfaces for rotary shaft seals $[14,15]$.

MBN is based on axial profile measurements. They are measured mostly tactile on the circumference of a shaft in defined angle intervals. The circumferential line up of the profiles results in the so-called "pseudo topography". This is a $3 \mathrm{D}$ topography with a much higher resolution in the axial direction than in the circumferential direction. As a next step, a Fast Fourier Transformation (FFT) captures dominant frequencies of the pseudo topography. Then, the dominant frequencies are summarized in an initial function based on harmonic cosine. The result of fitting the initial function to the measured profiles is a mathematically approximated surface. The real surface is evaluated by the parameters derived from this mathematical surface, which is why the measurement method is indirect and model-based in this sense. The current computation procedure of the MBN-method is based on the 2nd generation lead measurement developed by Seewig [16]. Further measuring methods for lead structures are for example summarized in [17].

The evaluation strategy of the MBN-method is well suited to detect strongly periodic structures on turned, rolled and grinded shafts. However, the frequency-based mathematical model always determines a periodic structure without a threshold for valid or non-valid results. It always assumes a circulating, periodic structure, even if it is not present in reality. Aperiodic and stochastically distributed structures cannot be described. Further, there is a need for optimization in the measurement procedure. The MBN-method covers two ranges of $360^{\circ}$ and $36^{\circ}$ on the shaft circumference with 72 axial profile measurements each. The loss of information between each measurement leads to a resolution in circumferential direction that is determined by the shaft diameter. It is significantly lower than the resolution in axial direction. Different results of both measuring grids, wrong and inconclusive lead patterns may occur because of the lower circumferential resolution. This requires a trained expert to interpret the evaluation results.

\subsection{Structure-based lead analysis based on optical measurement data}

Structure-based methods offer a different approach to the assessment of lead and have already been proven in the past, especially for micro lead. Baitinger [9] and Baumann [7] describe a structure-based method to analyse micro lead, target measurement strategies are examined in these studies $[7,18]$. Structure-based evaluation involves assessing the extent of a structure on the actual measured surface to determine individual structural elements. The geometries of the structures extracted in this way can all be described individually and then considered statistically. It is no longer necessary to mathematically approximate the real surface. Structure-based methods can thus overcome the described limitations of the frequency-based method for the evaluation of macroscopic lead.

The optical measurement data are particularly suitable as a data basis for structure-based evaluation methods. The advantage here lies not only in the high resolution of the measurement data, which is particularly useful in the detection of microscopic structures. Optical measured topographies use equidistant grids of measuring points with height information. They represent a structure on the topography in an equal scale in axial and circumferential direction, which is a prerequisite for the structure-based evaluation method. With tactile measuring methods, this is only possible with an enormous expenditure of time. Figure 3 summarizes the limitations of frequency-based methods and the resultant potential of a structure-based lead analysis. 


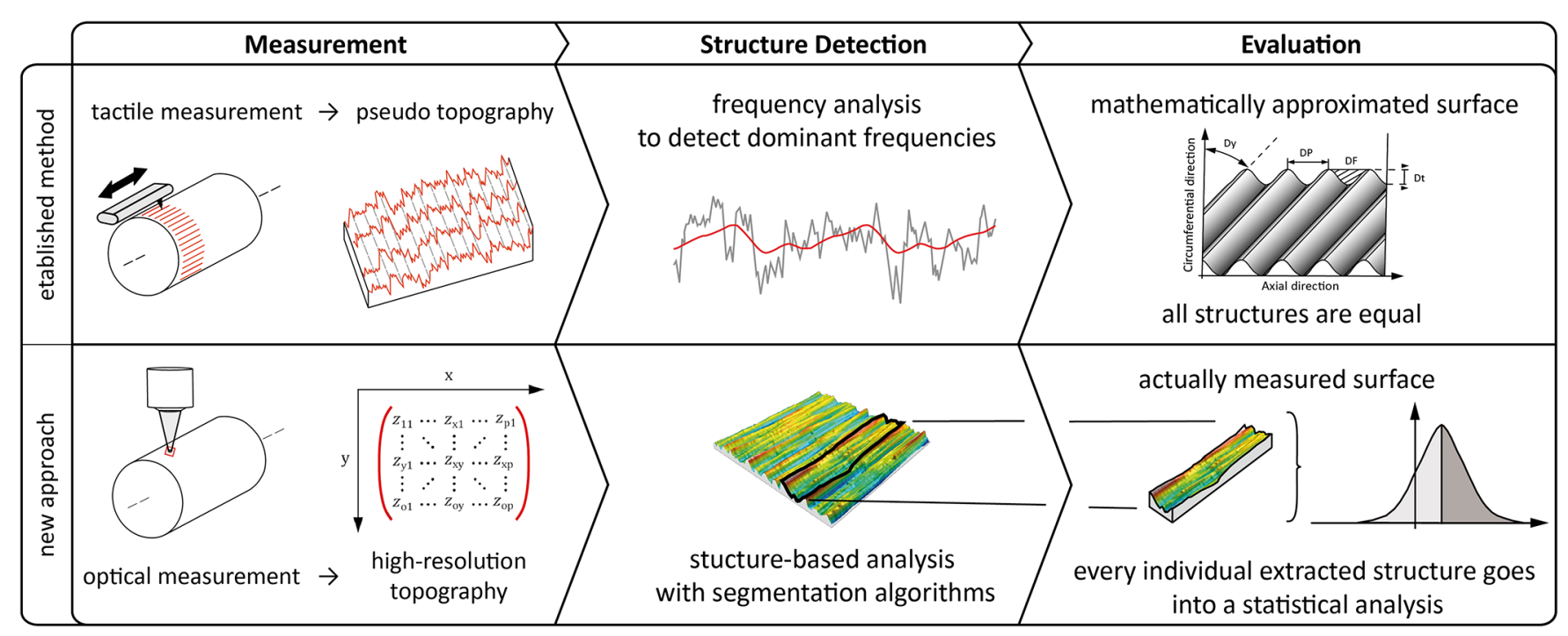

Fig. 3. Comparison of frequency-based and structure-based analysis.

On the one hand, the new evaluation method should enable the detection of periodic and circulating as well as aperiodic and stochastically distributed macroscopic lead structures. On the other hand, this procedure aims to provide a comprehensive analysis of the lead structures of all categories described in Figure 2. The measurement of the shaft surface on a single measuring instrument should be sufficient in the future to measure all types of lead. The data compatibility for the analysis of macro lead, micro waviness and micro lead is a prerequisite.

\subsubsection{Surface segmentation - watershed transformation and basic considerations}

With regard to the scope of application "lead in sealing technology", the term structure describes a self-contained depression in the surface. These types of structures contain fluid when in contact with the sealing edge and can act as flow channels when the shaft is rotating. In order to find such structures on technical surfaces, the basic idea of a study by Maxwell from the 19th century is used [19]. Maxwell found that landscapes can be divided in regions of dales and hills. These regions are delimited by watershed lines (for dales) and watercourse lines (for hills). Watersheds lines thus separate two dales, the flow of water starting from a peak flows through a dale along the watercourse lines to the local lowest point.

This has given rise to a segmentation method of digital image processing called watershed transformation (WST). Segmentation involves dividing an image into individual regions and isolating certain features of the image. The WST is mainly applied as a tool of mathematical morphology on grey scale images to extract features or to detect patterns [20]. With the most direct reference to its origin, the DIN EN ISO 25178-2 standard [21] describes the application of watershed transformation to 3D topographic measurement data. The surface segmentation by means of WST is the basis for the derivation of the feature parameters, with which, for example, the contact area of the friction partners of a tribological system can be represented. Blateyron describes in detail their derivation and various applications $[22,23]$. The standardization of a feature-based evaluation method for profiles is in progress [24].

According to the DIN EN ISO 25178-2 standard, the WST segments a topography into texture features which are grouped into "areal features (hills and dales), line features (course and ridge lines) and point features (peaks, pits and saddle points)" [21]. Peaks form the highest point of a hill, pits the lowest point of a dale. Saddle points result from the crossing points of course and ridge line or else watershed line. In this model conception, an imaginary rising water level would run out over the lowest saddle point of a dale.

The watershed transformation has been widely explored for segmentation of technical surfaces with respect to structural extraction, for example by Weidner [25] and Baumann [7]. In context to fluid pumping structures, a segmentation of the topography into dale regions is of interest. If a dale region is delimited by watershed lines sufficiently high and besides has a certain size, it can be regarded as a structure capable of containing and transporting a sufficiently large amount of fluid. The dale's shape can provide information on flow rates and flow direction.

Respectively, hill regions delimited by course lines can also be considered. Course lines also contain information about the pumping characteristics of the topography. They can be determined by applying the WST to the inverted topography. But watershed lines define the boundaries of a structure and a geometrically describable shape of this structure. Therefore, the segmentation into dale regions based on the Maxwell dale is pursued further here.

However, the result of the WST on topographic surface data is initially always over-segmented. This means that there are many small dale regions which have no significant influence on the fluid pumping on their own. Actually, they should be part of a larger, superior dale region. Measurement artefacts like process-related measurement noise partly cause the over-segmentation. They are unavoidable in optical measurements. Furthermore, the result of the WST shows real existing dales regions which are considered 


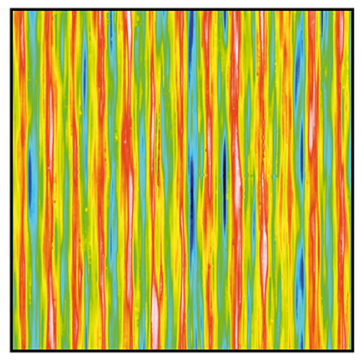

Topography

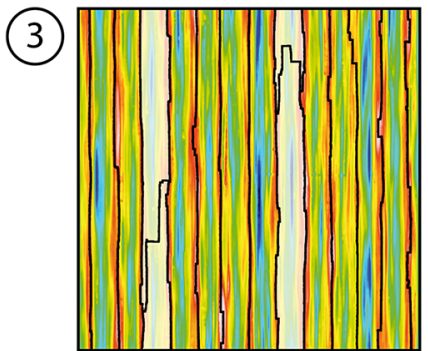

$30 \% \mathrm{Sz}$
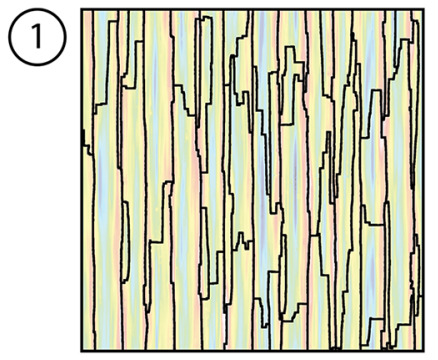

$10 \% \mathrm{Sz}$

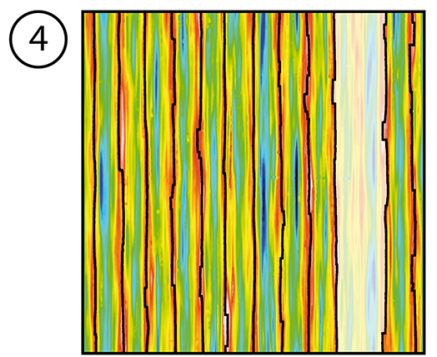

$40 \% \mathrm{Sz}$
(2)

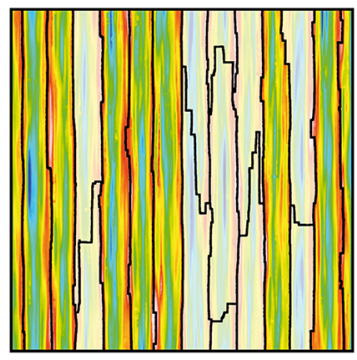

$20 \% \mathrm{Sz}$

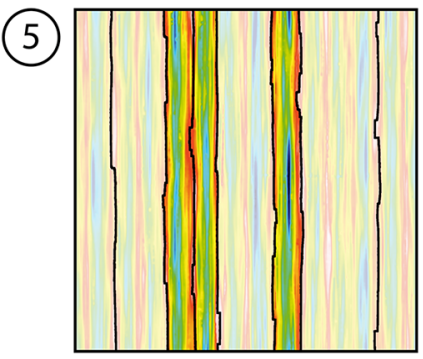

$50 \% \mathrm{Sz}$

Fig. 4. Application of WST + Wolf pruning with varying pruning heights.

to be over-segmented. To cut the over-segmentation, the dale regions must be systematically merged to larger regions. The DIN EN ISO 25178-2 standard therefore specifies a method for reducing the over-segmentation for use with surface topographies.

\subsubsection{Standardized method for reducing over-segmentation and suitability for lead structure capturing}

The standardized method in DIN EN ISO 25178 for reducing over-segmentation used in combination with the WST is the so-called Wolf pruning, as described by Wolf [26] and Scott [27]. It combines the areal features based on their relative heights. The heights are calculated from the difference of pits and its lowest saddle points, or peaks and its highest saddle point. For dale regions it works as follows: dale regions with a height below a defined threshold are integrated to a deeper dale region in the immediate vicinity. The direction of fusion is determined by the position of the lowest saddle point. By cutting the connection of watershed lines to the saddle point, a larger dale region is created. The threshold is calculated commonly as a percentage of the maximum height of the surface topography given by the $\mathrm{Sz}$ value [21]. A detailed description of the WolfPruning and other methods of reducing the oversegmentation are given in [28].

But, this procedure also has limitations that make it impractical for the extraction of lead structures. The $\mathrm{Sz}$ value describes the height difference between the highest and lowest measuring point of the entire topography. Its scale is usually much larger than the height of a lead structure on the topography. In addition, the Sz value is very susceptible to spikes and thus also dependent on the measurement method. This makes the results of this procedure highly dependent on the threshold set.
Small changes in the threshold value already affect the number and shapes of the extracted structures. Inhomogeneities of the surface and the large bandwidth of occurring structures prevent the definition of a generally valid threshold. Figure 4 illustrates this problem by means of the Wolf pruning results of a ground shaft surface with macro lead. Increasing the pruning height relative to the $\mathrm{Sz}$ value in 10 percentage point increments clearly shows the influence on segmentation result.

The degree of over-segmentation decreases steadily, but the clearly visible, continuous surface structure cannot be represented with any of the five results. Topography (3) with $30 \%$ of Sz still shows interruptions, but at topography (4) with $40 \%$ of Sz two supposedly separate structures are already combined.

More stable results can be achieved by region-specific threshold values. Furthermore, the consideration of pure amplitude values is not sufficient to judge whether the course of a structure continues or breaks off. Much more important than punctual height information of the structure itself is the connection of the structure to a neighbouring structure. Information about the connection contains the location and course of the respective watershed lines between them. The additional inclusion of this lateral information is the main component of the new approach, which is presented in chapter 3. Under these aspects, the functionality of watershed segmentation is extended by a new procedure specially developed for the counterfaces of rotary seals.

\section{New procedure for the reduction of over-segmentation}

The new approach aims to map fluid paths and flow directions. The procedure follows the scheme in Figure 5. 


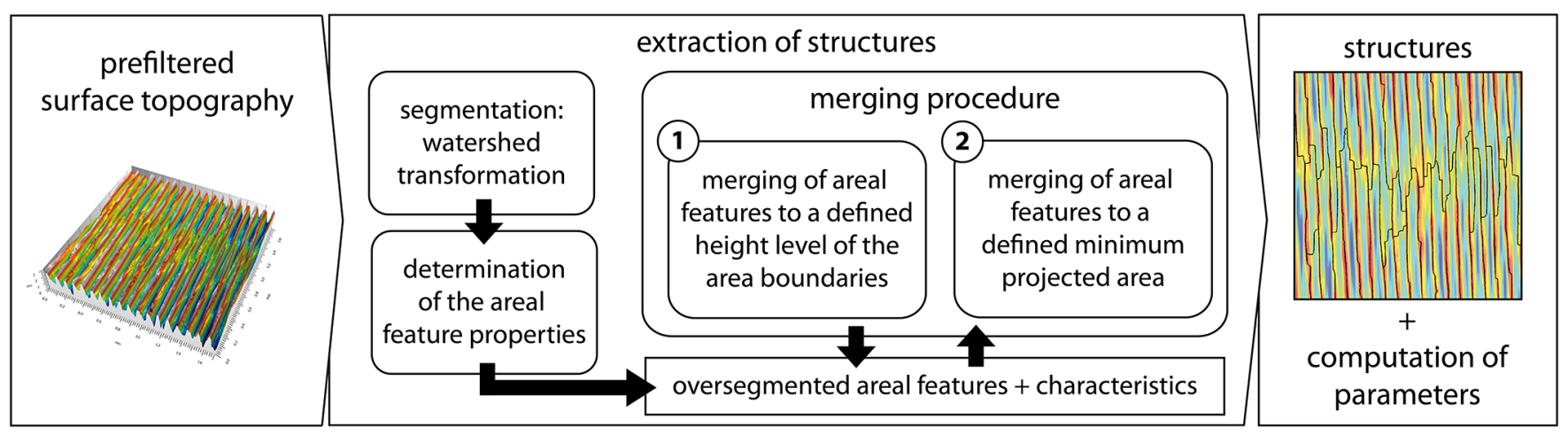

Fig. 5. Schematic sequence of the structure-based procedure.

As a first step to this goal, the topography is segmented into individual elements using the WST in its original form. The functional principle of the WST was implemented in an algorithm for this purpose. As a result of the segmentation, there is also an over-segmented topography at first. Besides to a pixelwise areal feature assignment, each boundary section $B_{i, j}$ of an areal feature $A_{i}$ is also determined. These boundary sections $B_{i, j}$ separate it from a specific other areal feature $A_{j}$. Accordingly, a boundary section can be assigned to each of two areal features that it separates. It is thus part of a watershed line. This forms the basis for the further merging of areal features. In contrast to the Wolf pruning, no point features are considered to determine this direction. The focus is on the line features. The segmentation procedure is illustrated in Figure 6 using the example of the segmented area $A_{1}$. The segmented areal feature $A_{1}$ is surrounded by seven neighboured areal features $A_{2}$ to $A_{8}$ and thus delimited by seven boundary sections $B_{1,2}$ to $B_{1,8}$.

The merging procedure after the segmentation with WST is achieved by the following explained strategy. The fluid passes an areal feature $A_{i}$ in the circumferential direction. The merging direction with another areal feature $A_{j}$ is determined by the direction of flow when the fluid exits the areal feature $A_{i}$. The boundary section between the areal feature and its neighbor areal feature acts as a gap which connects both. Accordingly, it must be determined whether a fluid flows into or out of the areal feature $A_{i}$ and over which boundary section $B_{i, j}$.

To determine the position of possible gaps, a further model representation is used. The fluid flows in the circumferential direction due to the rotation of the shaft. This flow can be deflected in its direction by elevations on the surface. Thus, the fluid would rather flow through a gap perpendicular to the circumferential direction and not parallel to it, because flow resistance is lower there. In order to weight this effect, the boundary sections are projected in circumferential direction. The height course is evaluated based on the projection. Figure 7 illustrates this with the boundary section $B_{1,2}$. To identify the gap's location, a segment of each boundary section is considered. This segment is marked red in Figure 7. Its projected length $l_{\text {seg, } p}$ represents the minimum width of a gap for the outflow of fluid, viewed in circumferential direction. The segment is moved along the boundary section until it is at its lowest height on average. The actual length of the segment $l_{\text {seg }}$ may change during movement, but never its projected length $l_{\text {seg, } p}$. If boundary sections have a less projected width than the defined projected segment length, all their pixels are included in the computation. The average height $c\left(l_{\text {seg,p } p}\right)_{\text {seg } i, j}$ of an identified segment is the characteristic value of the corresponding boundary section $B_{i, j}$.

To assess if and where a fluid will enter or leave the areal feature, a reference height level is established. This reference height level is called the reference outflow-level $c_{\text {refi }}$ and is assigned to each areal feature. The segments heights $c\left(l_{\text {seg,p }}\right)_{\text {segi,j }}$ of an areal feature $A_{i}$ refer to its reference outflow-level height $c_{\text {refi }}$ and must run above it, otherwise border sections running below it will be eliminated in the merging procedure 1 . Figure 8 illustrates merging procedure 1 and the derivation of the reference outflow-level height $c_{\text {ref }}$.

The height of a reference outflow-level $c_{r e f i}$ is determined in a delimited structure-seal contact zone $Z_{i}$ in which the associated areal feature $A_{i}$ is located. The determining of structure-seal contact zones $Z_{i}$ is the first step within one iteration loop of the merging procedure 1 . A structure-seal contact zone $Z_{i}$ is defined in its length so that it spans the areal feature $A_{i}$ in circumferential direction. Its width in axial direction corresponds to the contact band width $b$ of a rotary shaft seal. An assumed value for contact band width here is $b=0,15 \mathrm{~mm}$. The axial positioning of the structure-seal contact zone $Z_{i}$ is centred on the areal feature $A_{i}$. By creating these structure-seal contact zones, a sub-area is considered on the topography, which should reflect the contact surface of the seal. Figure 8 shows this on the left with an example areal feature. Inhomogeneities on the topography outside a structure-seal contact zone do not influence each other.

After the zoning, the functional volume parameters according to DIN EN ISO 25178-2 [21] are determined in each structure-seal contact zone $Z_{i}$. These are computed on the basis of the material ratio curve, shown in Figure 8, centre. The material ratio curve is of interest because the dale void volume $(V v v)$ and the core void volume $(V c)$ provide information about the fluid capacity in the structure-seal contact zone. Furthermore, the curve shows specific courses for certain surface properties. The height of a reference outflow-level $c_{\text {ref } i}$ is calculated depending on the cutting height of the dale $c(q)$ and the core $c(p)-c(q)$. 


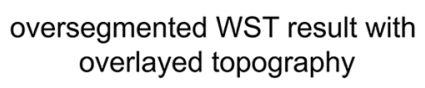

oversegmented WST result with overlayed topography

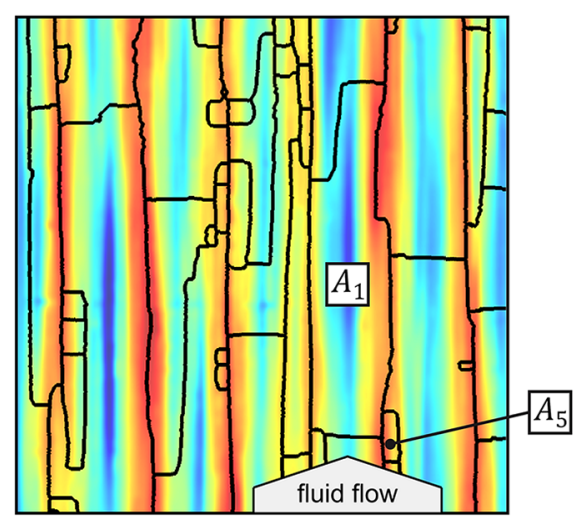

$A_{\mathrm{i}}$ areal feature

(B,i, boundary section

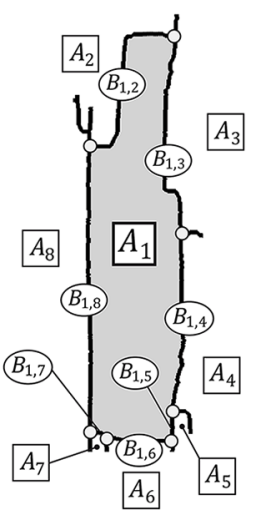

Fig. 6. Topography segmentation procedure.

A further parameter $x_{V m c}$ determines the height of a reference outflow-level $c_{r e f i}$ as a percentage of the core height according to equation (1).

$$
\mathrm{c}\left(x_{V m c}\right)_{r e f}=c(q)+x_{V m c} \cdot(c(p)-c(q)) .
$$

The elimination condition for a boundary section $B_{\text {limi,j }}$ derives from equation (2). This elimination condition applies to two boundary sections of the example in Figure 8, which can be seen on the right-hand side.

$$
B_{l i m i, j}=c\left(l_{\text {seg,p }}\right)_{\text {seg } i, j}<\mathrm{c}\left(x_{V m c}\right)_{r e f i} .
$$

After merging procedure 1, there are still over-segmented areal features which cannot be regarded as a separate structure. These areal features result from ambiguous boundary courses of a superordinate structure. Since their boundary sections are all at a high level, they are not eliminated by merging procedure 1 . Such an over-segmented areal feature is, for example, the areal feature $A_{5}$ in Figure 6. A commonality of the remaining over-segmented areal features is a small projected area $a_{\text {proj }}$ compared to the other areal features. In order to identify them, a reference value $a\left(x_{\text {alim }}\right)_{\emptyset, \text { proj }}$ is calculated according to Equation (3). This reference value represents a percentage $x_{\text {alim }}$ of the average projected area of all areal features $a_{\emptyset, p r o j}$. The percentage share $x_{a l i m}$ is an evaluation parameter to be defined. Still over-segmented areal features $A_{\text {lim } i}$ fulfil the condition in equation (4) and have a smaller projected area $a_{\text {proji }}$ than the reference value $a\left(x_{a l i m}\right)_{\emptyset, p r o j}$ specifies.

$$
\begin{gathered}
a\left(x_{\text {alim }}\right)_{\emptyset, \text { proj }}=x_{a l i m} \cdot a_{\emptyset, \text { pro } j} \\
A_{\text {limi }}=a_{\text {proji } i}<a\left(x_{a l i m}\right)_{\emptyset, p r o j} .
\end{gathered}
$$

By eliminating their boundary section with the lowest height of the determined projected segment, these areal features are integrated into a neighbouring superordinate

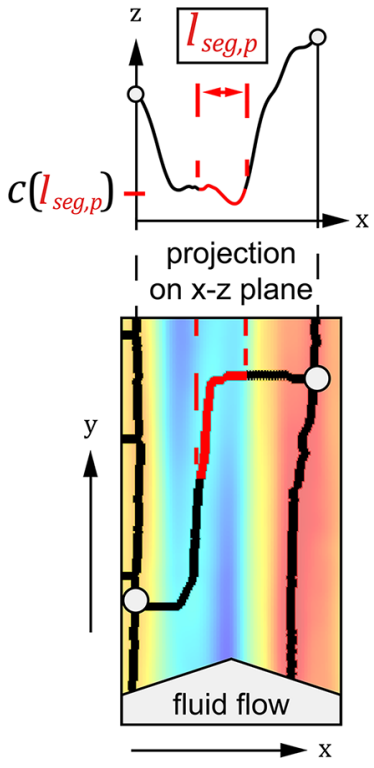

Fig. 7. Determination of the boundary section segment.

structure. This second merging procedure also runs through an iterative loop. It stops when all areal features have reached the certain size to each other. They can then be considered as a structure. Figure 9 describes the overall merging procedure with a schematic sequence of the steps. The result is the main structure extracted from the topography.

For each extracted structure, its geometric shape can then be described. This is done with six parameter classes for each structure. For the structure $i$ of a total of $n$ structures, these parameters are the structure orientation $\mathrm{SD} \gamma_{i}$, the structure width $\mathrm{SDB}_{i}$, the structure depth $\mathrm{SDT}_{i}$, the structure length $\mathrm{SDL}_{i}$, the structure cross section $\mathrm{SDF}_{i}$ and the structure volume $\mathrm{SDV}_{i}$. The designation and its computation are explained in Figure 10.

The structure-based evaluation method now allows to look statistically at the individual parameter values. For this purpose, the mean values, median values and standard deviations are computed for each class. The mean value and the median value describe the average structure of a topography, whereas the median is less susceptible to outliers. The dispersion of the values per parameter class is described by the standard deviation.

\section{Results and analysis}

Three different ground shaft surfaces are used to illustrate the presented procedure. The measurement data was recorded with a confocal topography measuring instrument. The 3D topographies consist of stitched single measurements and have an overall measuring field size of $1.17 \mathrm{~mm} \times 1.17 \mathrm{~mm}$. The resolution of the measurement data matrix is scaled to $1 \mu \mathrm{m}$ for the investigations. The topographies are subjected to common surface filters. They are illustrated threedimensionally in Figure 11. 
determining of structure seal contact zones $Z_{i}$

elimination of boundary sections
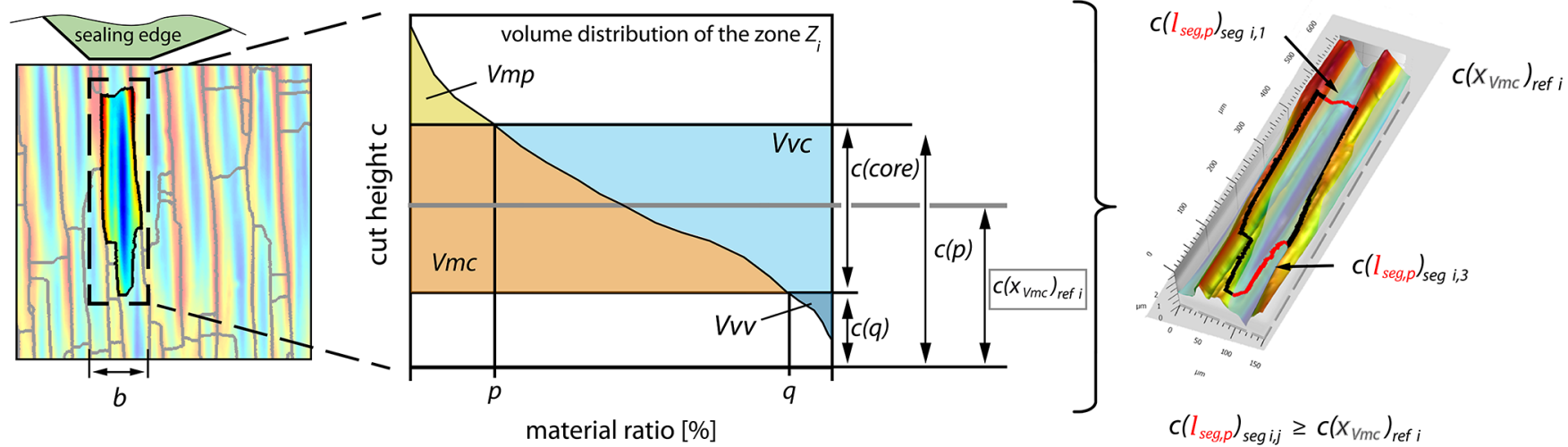

\begin{tabular}{|cl|}
\hline analysis parameters \\
\hline$x_{V m c}$ & percentage of the core height c(core) \\
$l_{\text {seg,p }}$ & projected length of a segment \\
$b$ & contact band width of rotary shaft seal and shaft surface \\
\hline
\end{tabular}

\begin{tabular}{|ll|}
\hline reference values \\
\hline$c\left(l_{\text {seg,p }}\right)_{\text {seg }}$ & $\begin{array}{l}\text { average height of a segment of the } \\
\text { projected length } l_{\text {seg,p }} \\
\text { height of the reference outflow-level as a } \\
\text { percentage of the core height } x_{V m c}\end{array}$ \\
\hline
\end{tabular}

\begin{tabular}{|cc|}
\hline \multicolumn{2}{|c|}{ functional volume parameters } \\
\hline$V v v$ & dale void volume \\
$V v c$ & core void volume \\
$V m c$ & core material volume \\
$V m p$ & peak material volume \\
$p$ & upper material ratio (80\%) \\
$q$ & lower material ratio (10\%) \\
\hline
\end{tabular}

Fig. 8. Derivation of the reference outflow-level $c_{r e f}$.

over-segmented topography section

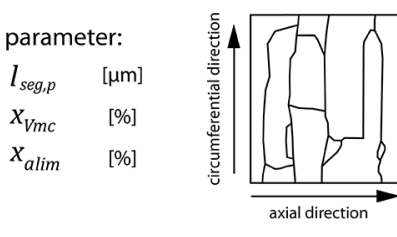

legend:
$A_{\mathrm{i}}$ areal feature
$S_{\mathrm{i}}$ structure
(Bi, border section
$\left\ulcorner Z_{\mathrm{i}}\right\lrcorner$ zone
- lowest continous segment of the projected length $l_{\text {seg,p }}$

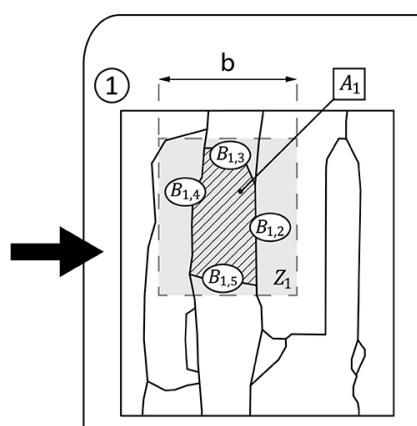

(2)

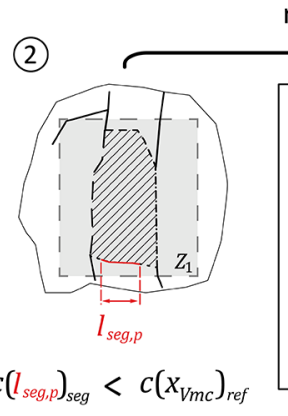

merging procedure 1
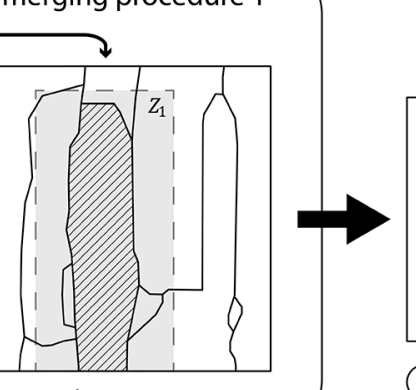

(3)

(4)

merging procedure 2

extracted structures

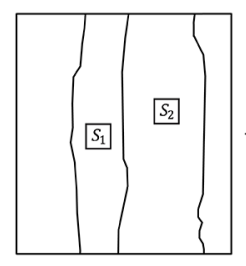

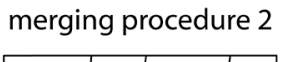
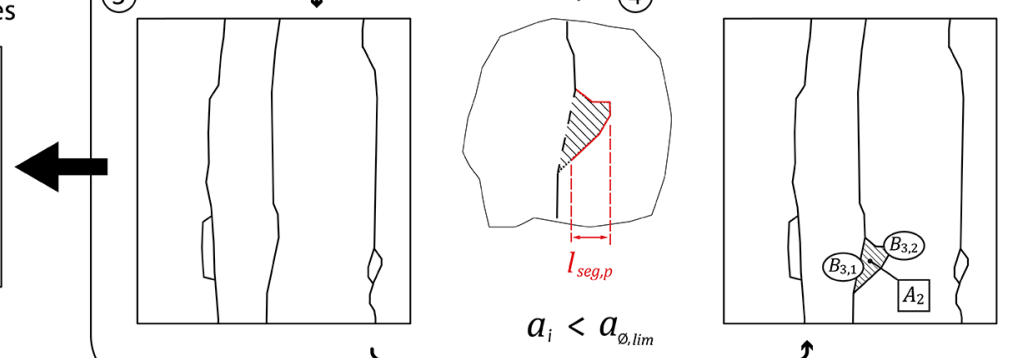

Fig. 9. Merging procedure to extract lead structures of over-segmented topographies. 


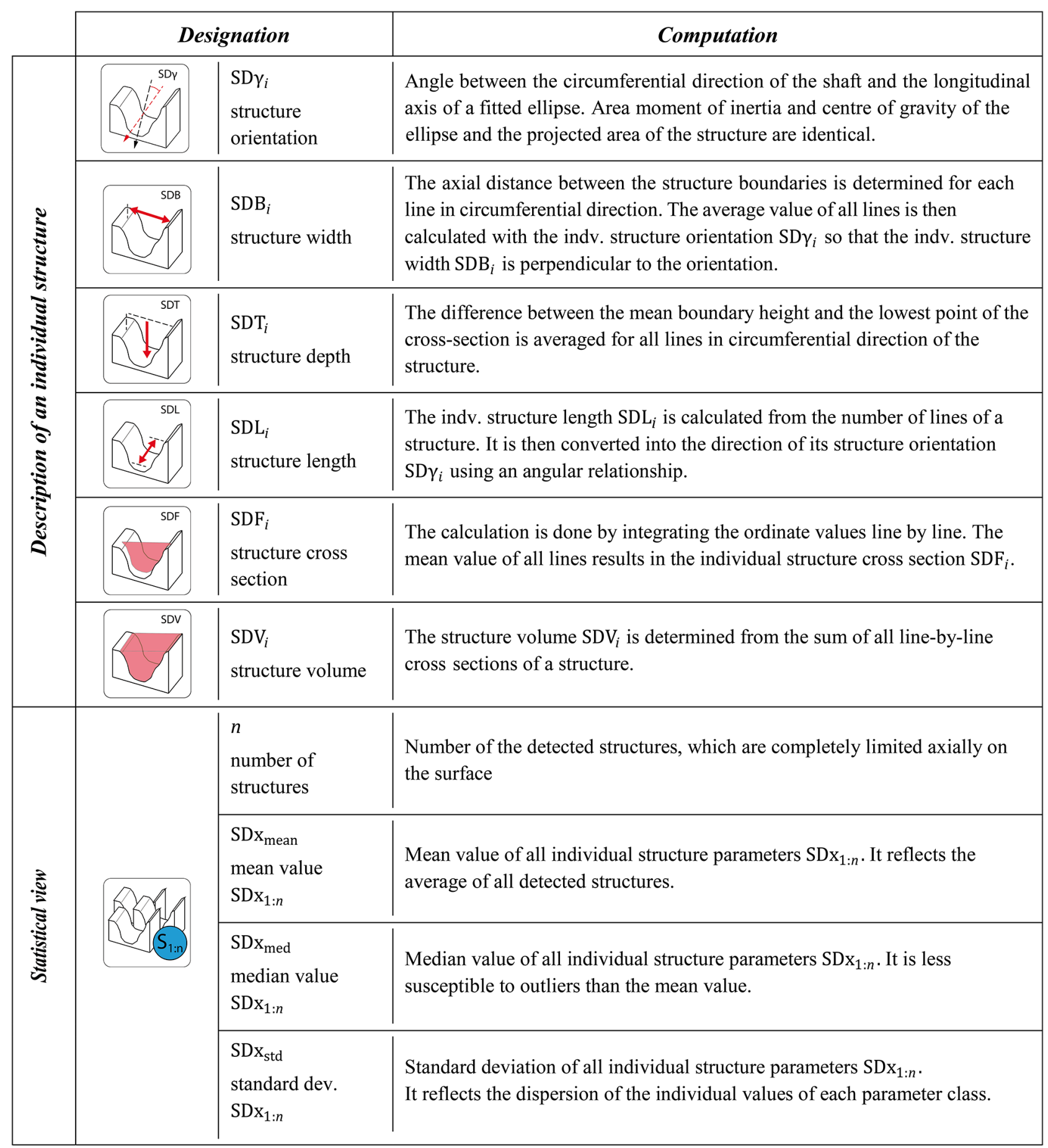

Fig. 10. Structure-based parameters for the description of lead. 
In addition, the results of the MBN-method are presented over the $360^{\circ}$-range and the $36^{\circ}$-range. It shows the measured pseudo topography above and the mathematically approximated surface in the middle. One of the 72 axial profiles (green) is shown below, overlaid with the mathematical lead surface (black). The associated lead parameters according to MBN 31007-7 are also listed for both measuring ranges.

The selection of the investigated surfaces (see Fig. 11) was based on the following characteristics in relation to the results of the MBN-method:

- Surface 1 shows an obvious macro lead with a continuous structure on both the 3D topography and the pseudo topographies. The MBN-method provides meaningful results here.

- On the 3D topography of surface 2, no delimited dales are visible, only non-contiguous elevations. The MBNmethod cannot detect any significant periodicity on both pseudo topographies. This can be seen in the low match between the profile of the mathematical lead surface and the roughness profile. The amplitude of the initial function is therefore low compared to the surface's roughness. Although the mathematical surface and the lead parameters derived from it do not reflect reality at all, but they correspond to the result of a macro lead-free surface.

- Delimited dales can be recognized on 3D topography of surface 3 , even if their boundaries are not as evenly pronounced as on the 3D topography of surface 1 . Some structural boundaries run into each other or fluctuate in height. This phenomenon causes different results of the MBN-method for the measurements over a range of $360^{\circ}$ and $36^{\circ}$. The resolution in the circumferential direction of the $360^{\circ}$ pseudo topography is not sufficient to depict the structures. A more meaningful representation of the surface can be achieved with the $36^{\circ}$ pseudo topography. This shows the better match of the profile of the mathematical lead surface with the roughness profile. However, the extent to which these structures can be considered as continuous macro lead structures is difficult to judge on the basis of these results.

Figure 12 presents the results of the structure-based analysis in the lower section. The structure-based analysis was carried out for the three surfaces with the same parameterisation, also listed in Figure 12. For illustration purposes, the topographies are overlaid with the result's structure boundaries in the plan view. Here, the structurebased statistical parameters which characterise the surfaces are also listed. A graphical comparison of the statistical results of the three surfaces is given in Figure 13. A discussion of the results and a comparison with the MBN-method's results follows.

On surface 1 , the determined course of the structures corresponds to the subjective expectation. The structurebased parameters and their corresponding lead parameters according to MBN 31007-7 show a good consistence, as can be seen in Figure 13. The structure length SDL and the structure volume SDV have no counterpart. The structure-based evaluation now provides completely new evaluation criteria at this point. For example, the average structure length $\mathrm{SDL}_{\text {mean }}=1172.0 \mu \mathrm{m}$ is equal to the length of the measuring field in the circumferential direction. This characteristic points to a continuous course of the structures. The low dispersion relative to the mean value of all parameters reflects a high similarity of all extracted structures and an equal orientation. In summary, structurebased and MBN-method's results agree because the real surface shows a strong periodic structure.

The extracted structures of surface 2 are subjectively difficult to comprehend, but the statistical consideration of the individual parameters provides meaningful statements about the lead-free sealing counterface. The structures fit between elevations of the surface and occur in most diverse size. A preferred direction of the structures cannot be determined. This is elucidated by the median value of the structural orientation $\mathrm{SD} \gamma_{\text {med }}=-0.013^{\circ}$, which is close to the zero-angle position, as well as the wide range of orientation specified by the comparatively large standard deviation $\mathrm{SD} \gamma_{\mathrm{std}}=1.383^{\circ}$ around the mean value $\mathrm{SD} \gamma_{\text {mean }}=0.113^{\circ}$. The large standard deviation of the individual structure widths $\mathrm{SDB}_{\text {std }}=19.22 \mu \mathrm{m}$ in relation to its mean value $\mathrm{SDB}_{\text {mean }}=51.53 \mu \mathrm{m}$ also indicates no regularity in the distribution of the structures in the axial direction. For this reason, there is no correspondence with the period length of the surface $\operatorname{DP}\left(360^{\circ}\right)=\operatorname{DP}\left(36^{\circ}\right)=$ $93 \mu \mathrm{m}$ and the structure-based determined structure widths $\mathrm{SDB}_{\text {mean }}$ or $\mathrm{SDB}_{\text {med }}$. Figure 13 shows that this clear difference is also present in the lead depth Dt and its structure-based counterparts because a periodicity as recognized by the FFT does not exist on the real surface. The term aperiodic surface without macroscopic lead structures can be aptly applied for surface 2 .

The structure-based analysis of surface 3 partially confirms the result of the MBN-method of the $36^{\circ}$ measurement, well visible in Figure 13. Although the scatter of the structure angles with $\mathrm{SD} \gamma_{\mathrm{std}}=0.858^{\circ}$ is significantly larger than for surface $1\left(\mathrm{SD} \gamma_{\mathrm{std}}=0.237^{\circ}\right)$, the structures statistically show a clear preferred direction deviating from the circumferential direction. This results in an agreement between the values of lead angle $\mathrm{D} \gamma\left(36^{\circ}\right)=$ $-0.328^{\circ}$ and the median $\mathrm{SD} \gamma_{\mathrm{med}}=-0.294^{\circ}$. Median $\mathrm{SDB}_{\text {med }}=61.39 \mu \mathrm{m}$ and mean $\mathrm{SDB}_{\text {mean }}=61.73 \mu \mathrm{m}$ of the structure widths are almost equal and close to the value of the period length $\operatorname{DP}\left(36^{\circ}\right)=65.0 \mu \mathrm{m}$. The small standard deviation of the individual structure widths $\mathrm{SDB}_{\text {std }}=7.12 \mu \mathrm{m}$ reflects an axially periodic distribution of the structures. The other parameters show larger standard deviations compared to surface 1 with strong periodic macro lead. This is because in addition to continuous structures, interrupted structures are also detected on the surface 3 . Thus, a directed micro waviness is detected, which is highly probable to be harmful to sealing in the case of a rotary shaft seal. However, a pumping effect comparable to a structure that continuously circulate the shaft's diameter is not to be expected.

Overall, it can be said that sealing counterfaces with and without lead structures can be described much more realistically with the structure-based approach. Realistic here means that the parameters have a direct relation to 


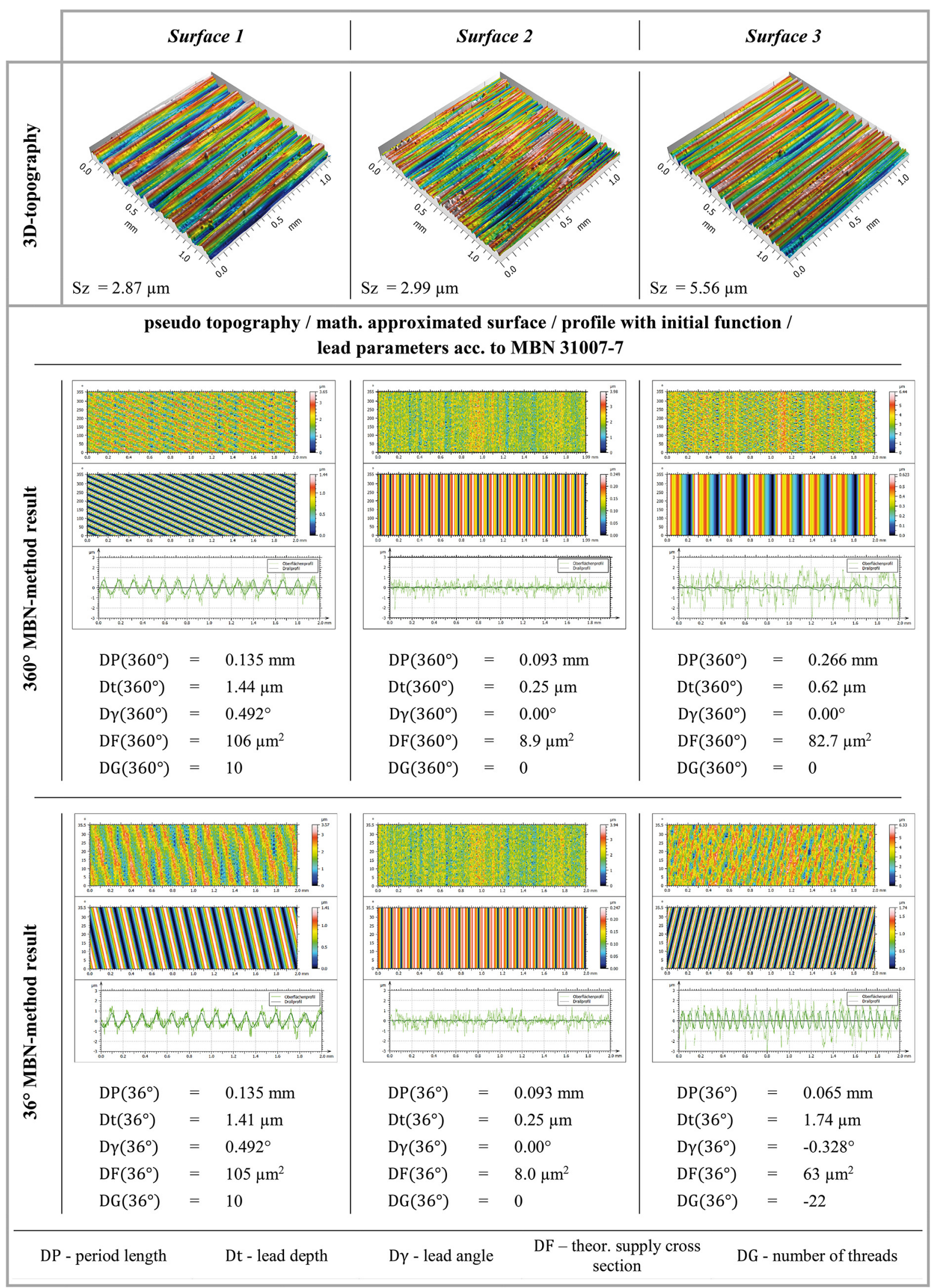

Fig. 11. Investigated surfaces - optical measured topographies in the $3 \mathrm{D}$ view, $360^{\circ}$ and $36^{\circ}$ results of the MBN-method. 


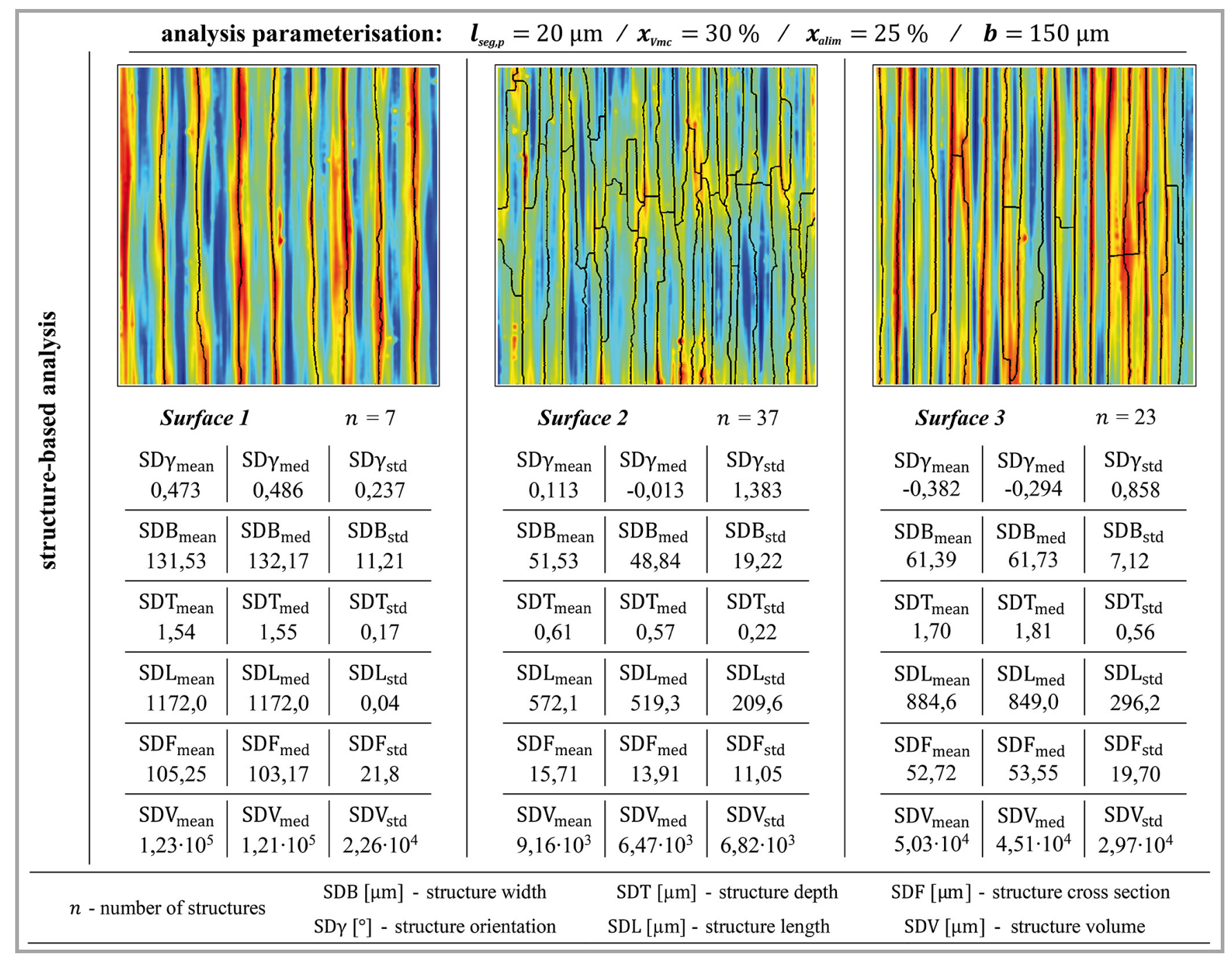

Fig. 12. Investigated surfaces - structure-based analysis results.

the measured surface. Even for surfaces with strongly periodic structures like surface 1, the lead depth of the mathematical surface according to MBN 31007-7 is always smaller than the structure-based counterpart because of the approximation. If a periodicity on the surface is less pronounced or not present at all, only the structure-based approach is suitable to describe the lead structures on the shaft surface.

The presented structure-based method uses the advantages of optical measurements over tactile measurements, but optical measurements also bring disadvantages. The data of optical measuring systems always show spikes and invalid measuring points and, due to the principle, measurement noise. The extent of the measurement artefacts depends on the measurement method, the measurement instrument and the optical lenses used. Therefore, the merging procedure may provide different segmentation results for the same surface when measured on different optical measuring instrument. A measuring instrument or lens-dependent processing of the measurement data requires a high effort. In addition, not every optical measuring instrument or lens can be used to generate data because a high quality of the measurement data is required. Another limitation are surfaces, e.g. with coatings, that are not suitable for optical measurement and therefore cannot be evaluated.

Further research work also concerns the parameterisation of the evaluation algorithm. The implementation of the merging procedure requires the specification of parameters that influence the final result. The parameter setting is currently based on a plausibility check of the results according to the current state of knowledge about suitable and unsuitable sealing counterfaces. In this respect, simulation studies and experimental test runs, such as pumping rate investigations of the sealing counterface, must be carried out. The parameterisation of the evaluation algorithm must then be adapted in such a way that the evaluation results correlate as best as possible with the functional behaviour of a radial shaft seal. 

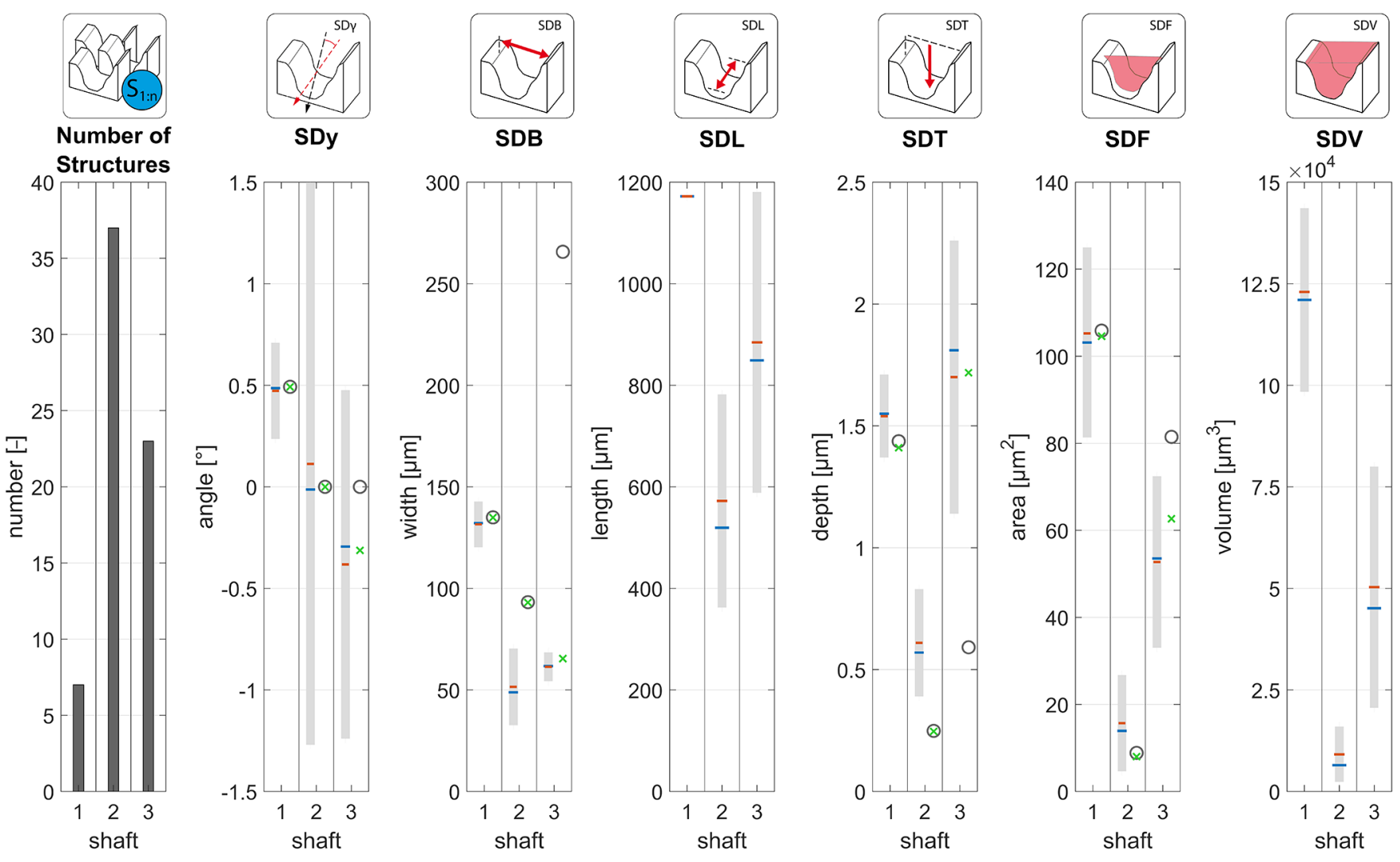

Structure-based : - Median Value

- Mean Value

Frequency-based : $\quad \mathrm{MBN} 360^{\circ}$

$\times \operatorname{MBN} 36^{\circ}$

Fig. 13. Comparison of the statistical structure-based parameters and MBN-method's results.

\section{Conclusion}

A new approach for the analysis of macroscopic lead structures is presented in this paper. The structurebased method pursues the extraction of structures capable of pumping in the sealing contact on optically measured 3D topographies. The structures are determined by tracing the flow paths of a fluid on the surface. This offers great potential for the analysis of macroscopic lead structures. Besides periodic macro lead, the detection of structures of different width and length is possible. These types of structures are described by the micro waviness which shall be included in the lead analysis in the future.

For the extraction of lead structures, the topography is first segmented into areal features. A segmentation algorithm according to the watershed transformation [21,29] is used for this. Its result is always an over-segmented topography. This forms the basis of a new procedure for merging the areal features. The strategy behind this is to match the merging direction of areal features with the outflow direction of a passing fluid. The areal features unite thereby to evaluation-relevant structures.

As a next step, the shapes of each detected structures on the surface are described by parameters. These parameters were derived by the segmented results for each individual structure. The surface is then classified by a statistical consideration of the individual structure parameters. This results in the statistical parameters mean value, median value and standard deviation of each parameter class. Results of the structure-based evaluation method were presented in this paper using three shaft surfaces with different surface characteristics. The results were also contrasted and compared with the results of the macro lead measurement according to the MBN 31007-7 [11].

Further potential exists in the consideration of the distribution diagrams and including different types of distribution. This raises also the question of measuring field size, number of measuring fields and the measuring grid. These factors directly influence the number of structures extracted and thus the statistical quality of the result. The aim is a sufficient statistical prediction of the surface quality.

With regard to the application "lead on sealing counterfaces", the presented method aims at more robust and parameter-independent results than standardized method for reducing the over-segmentation caused by the watershed transformation, the Wolf pruning according to DIN EN ISO 25178-2 [21]. For the three ground shaft surfaces presented, valid results of the structure-based evaluation are obtained with the same parameterisation. This raises the question of the smallest size of a macroscopic lead structure above which it can be considered stand-alone. The coarseness of the extracted structures is determined by the parameterisation of the merging algorithm. With the help of simulations and experimental test runs, an optimal parameterisation is to be determined. Correlation studies of the new measurement 
results with the functional behaviour of a rotary shaft seal must also prove the suitability of the structure-based method and the advantages over existing methods.

\section{References}

1. Deutsches Institut für Normung e.V.: DIN 3760, RadialWellendichtringe (Deutsches Institut für Normung e.V., Berlin, September 1996)

2. Deutsches Institut für Normung e.V.: DIN 3761-1, RadialWellendichtringe für Kraftfahrzeuge - Begriffe; Maßbuchstaben; zulässige Abweichungen; Radialkraft (Beuth, Berlin, January 1984)

3. International Organization for Standardization: ISO 6194-1, Rotary shaft lip-type seals incorporating elastomeric sealing elements - Part 1: Nominal dimensions and tolerances (2007)

4. F. Bauer, Federvorgespannte-Elastomer-Radial-Wellendichtungen (Springer Fachmedien Wiesbaden, Wiesbaden, 2021)

5. E. Prem, R. Vogt, Der Simmerring - Grundlagen zur Schadensprävention (Firmenschrift, Freudenberg Simrit GmbH \& Co. KG, Weinheim, 2008)

6. C. Fehrenbacher, W. Haas, 3D-Kennwerte. 3D-Oberflächenkennwerte für Dichtflächen. Abschlussbericht FVA Vorhaben Nr. 674 I, Frankfurt am Main: FVA, 2015, Forschungsvereinigung Antriebstechnik e.V. (FVA)

7. M. Baumann, Abdichtung drallbehafteter Dichtungsgegenlaufflächen - Messung, Analyse, Bewertung und Grenzen. Dissertation, Universität Stuttgart, Institut für Maschinenelemente, 2017

8. M. Baumann, F. Bauer, W. Haas, Messung, Analyse und Bewertung von Dichtungsgegenlaufflächen für das TriboSystem Radial-Wellendichtung, in 18th International Sealing Conference (ISC), Stuttgart, Germany, October 8-9, 2014; Frankfurt am Main: VDMA Fluidtechnik, 2014, pp. $627-639$

9. G. Baitinger, Multiskalenansatz mit Mikrostrukturanalyse zur Drallbeurteilung von Dichtungsgegenlaufflächen. Dissertation, Universität Stuttgart, Institut für Maschinenelemente, 2011

10. Deutsches Institut für Normung e.V.: DIN EN ISO 4288, Geometrische Produktspezifikation (GPS) - Oberflächenbeschaffenheit: Tastschnittverfahren; Regeln und Verfahren für die Beurteilung der Oberflächenbeschaffenheit, Beuth, April 1998

11. Mercedes-Benz: Norm MBN 31007-7, Geometrische Produktspezifikationen (GPS) - Oberflächenbeschaffenheit Mess- und Auswerteverfahren zur Bewertung von drallreduzierten dynamischen Dichtflächen (2008)

12. M. Schulz, M. Baumann, F. Bauer, Influence of different shaft surface finishes on the lubrication and friction condition of elastomeric lip seals, in 7th International Conference Integrity-Reliability-Failure, Funchal/ Portugal, 6-10 September 2020, 2020, pp. 351-368
13. S. Thielen, P. Breuninger, H. Hotz, C. Burkhart, T. Schollmayer, B. Sauer, S. Antonyuk, B. Kirsch, J.C. Aurich, Improving the tribological properties of radial shaft seal countersurfaces using experimental micro peening and classical shot peening processes, Tribol. Int. 155, 106764 (2021)

14. F. Bauer, M. Baumann, W. Haas, Scratch \& failure detection method for shaft and rod surfaces, in 16th Nordic Symposium on Tribology, Aarhus, Denmark, June 2014, 2014

15. F. Bauer, M. Baumann, W. Haas, Automated failure detection for sealing surfaces, in Nordtrib 2016, Hämeenlinna, Finnland, 14-17 June, 2016, 2016

16. J. Seewig, T. Hercke, 2nd generation lead measurement, in XIX IMEKO World Congress, Lisbon, Portugal, September 6-11, 2009, 2009

17. M. Baumann, G. Baitinger, F. Bauer, W. Haas, How to measure lead in sealing technology, Seal. Technol. 8-12 (2013)

18. P. Arnecke, J. Seewig, A Strategy for Micro-Twist Characterization on the Shaft in a Rotary Shaft Sealing System, in 18th International Sealing Conference (ISC), Stuttgart, Germany, October 8-9, 2014 (VDMA Fluidtechnik, Frankfurt am Main, 2014), pp. 653666

19. J.C. Maxwell, L. On hills and dales, London, Edinburgh, Dublin Philos. Mag. J. Sci. 40, 421-427 (1870)

20. J.B. Roerdink, A. Meijster, The watershed transform: definitions, algorithms and parallelization strategies, Fundam. Inform. 41, 187-228 (2000)

21. Deutsches Institut für Normung e.V.: DIN EN ISO 25178-2, Geometrische Produktspezifikation (GPS) - Oberflächenbeschaffenheit: Flächenhaft; Teil 2: Begriffe und OberflächenKenngrößen: Beuth, 17.040.30. September 2012

22. F. Blateyron, The areal feature parameters, in Characterisation of Areal Surface Texture, R. Leach, (Springer, Berlin, Heidelberg, 2013)

23. X. Jiang, N. Senin, P.J. Scott, F. Blateyron, Feature-based characterisation of surface topography and its application, CIRP Ann. (2021)

24. J. Seewig, P.J. Scott, M. Eifler, B. Barwick, D. Hüser, Crossing-the-line segmentation as a basis for Rsm and Rc Evaluation, Surf. Topogr. 8, 24010 (2020)

25. A. Weidner, Strukturorientierte dreidimensionale Rauheitsauswertung von optisch vermessenen Zylinderlaufbahnen (Shaker Verlag, Aachen, 2007)

26. G. Wolf, A fortran subroutine for cartographic generalization, Comput. Geosci. 17, 1359-1381 (1991)

27. P.J. Scott, Foundations of topological characterization of surface texture, Int. J. Mach. Tools Manufact. 38, 559-566 (1998)

28. G.W. Wolf, Scale independent surface characterisation: Geography meets precision surface metrology, Precis. Eng. 49, 456-480 (2017)

29. R. Leach, Characterisation of areal surface texture (Springer, Berlin, 2013)

Cite this article as: Maximilian Engelfried, Matthias Baumann, Frank Bauer, Three-dimensional structure-based approach for the analysis of macroscopic lead structures on sealing counterfaces, Int. J. Metrol. Qual. Eng. 13, 2 (2022) 